\title{
Charged Ferroelectric Domain Walls for Deterministic ac Signal Control at the Nanoscale
}

\author{
Jan Schultheiß,* Erik Lysne, Lukas Puntigam, Jakob Schaab, Edith Bourret, Zewu Yan, Stephan Krohns, \\ and Dennis Meier*
}

Cite This: Nano Lett. 2021, 21, 9560-9566

Read Online

ACCESS | Lill Metrics \& More | 国 Article Recommendations | st Supporting Information

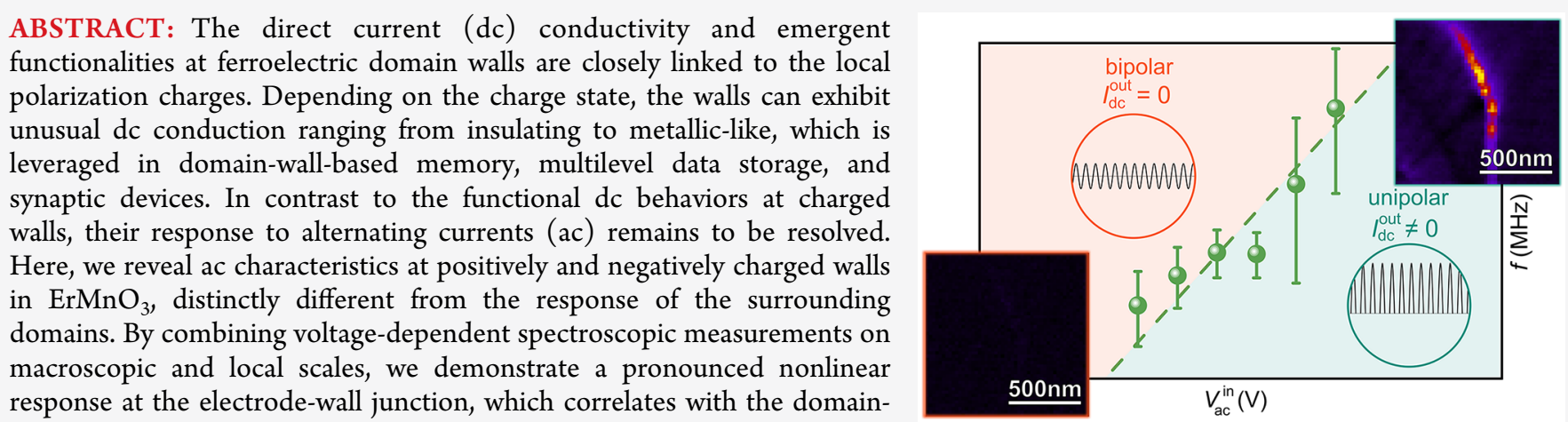
wall charge state. The dependence on the ac drive voltage enables reversible switching between uni- and bipolar output signals, providing conceptually new opportunities for the application of charged walls as functional nanoelements in ac circuitry.

KEYWORDS: Ferroelectric, domain walls, nanoelectronics, improper ferroelectricity, alternating current

\section{INTRODUCTION}

Ferroelectric domain walls are excellent candidates for the development of next-generation nanoelectronics, exhibiting a thickness that approaches the unit cell level. ${ }^{1-3}$ Similar to $2 \mathrm{D}$ systems such as graphene, ${ }^{4} \mathrm{MoS}_{2}$ single layers, ${ }^{5}$ and the $\mathrm{LaAlO}_{3} / \mathrm{SrTiO}_{3}$ heterointerface, ${ }^{6}$ they display unique electronic transport properties ${ }^{3}$ and large carrier mobilities. ${ }^{7}$ In addition to their transport properties, the ferroelectric domain walls are spatially mobile and can be injected and deleted on demand, which enables them to take an active role as reconfigurable elements in, for example, memory, ${ }^{8,9}$ diode $^{10}$ or memristor ${ }^{11}$ devices. Recently, it was demonstrated that intrinsic electronic correlation phenomena at ferroelectric domain walls can be used to control electrical currents, removing the need to write and erase the walls. ${ }^{12,13}$ This observation promoted the idea to develop the walls themselves into devices instead of using them as active elements in much larger electronic components. The approach is intriguing as it breaks the mold of classical device architectures, taking full advantage of the ultrasmall feature size of ferroelectric domain walls. Compared to more than a decade of research on domain-wall devices that operate based on the injection and deletion of domain walls, ${ }^{14,15}$ little is known about the technological potential of stationary walls. Only recently, it was shown that ferroelectric domain walls can be used to emulate the behavior of electronic components at the nanoscale, acting as binary switches ${ }^{12}$ and half-wave rectifiers. ${ }^{13}$ First insight into the electronic properties of domain walls under alternating currents (ac) was obtained for neutral domain walls in the gigahertz regime ${ }^{16-20}$ and applications as tunable microwave devices and acoustic wave filters have been suggested. ${ }^{21}$ In contrast, charged domain walls, which exhibit unusual conduction properties under direct current $(\mathrm{dc})$, have been found to be electronically inactive at high frequencies in the gigahertz regime. ${ }^{16,22}$

In this Letter, we study the electronic response at positively and negatively charged ferroelectric domain walls at intermediate frequencies in the kilo- and megahertz regime. Performing nanoscale spectroscopic measurements on $\mathrm{ErMnO}_{3}$, we observe domain-wall specific cutoff frequencies, $f_{\mathcal{C}}$ at which the current-voltage characteristic of the electrodewall junction changes from asymmetric to symmetric. By varying the ac voltage amplitude applied to negatively charged walls, we show that the cutoff frequency can readily be tuned by about 1 order of magnitude. This tunability enables

Received: August 17, 2021

Revised: October 21, 2021

Published: November 4, 2021 
reversible switching between uni- and bipolar output signals, facilitating active signal conversion in ac circuits at the nanoscale.

\section{RESULTS AND DISCUSSION}

ac Response of Positively and Negatively Charged Walls. Hexagonal $\mathrm{ErMnO}_{3}$ is a ferroelectric narrow band gap semiconductor (p-type, $E_{\text {gap }} \approx 1.6 \mathrm{eV}$ ). ${ }^{23-25}$ The spontaneous polarization is parallel to the $c$-axis $\left(P \approx 6 \mu \mathrm{C} / \mathrm{cm}^{2}\right)^{26}$ and originates from a structural lattice-trimerization, ${ }^{27,28}$ leading to explicitly robust ferroelectric domain walls, including all fundamental types of $180^{\circ}$ walls (i.e., neutral side-by-side walls, positively charged head-to-head walls, and negatively charged tail-to-tail walls). ${ }^{29}$ The conduction of the neutral walls has been intensively investigated both in the $\mathrm{dc}^{29-31}$ and $\mathrm{ac}^{13,16}$ regimes continuously covering frequencies up to the gigahertz range, and their basic electronic properties are well understood. In contrast, at charged domain walls only the dc transport behavior ${ }^{29,31,32}$ and the response at high frequencies in the microwave range ${ }^{16}$ have been studied, whereas their ac properties at intermediate frequencies remain to be explored.

The electrical dc transport of a (110)-oriented $\mathrm{ErMnO}_{3}$ single crystal (in-plane polarization) is displayed in the conductive atomic force microscopy (cAFM) map in Figure 1a. The orientation of the ferroelectric polarization is indicated by the arrows, determined from the calibrated piezoresponse force microscopy (PFM) image displayed in the inset of Figure 1a. The data shows the established transport behavior, ${ }^{29}$ that is, enhanced conductance (bright) at the tail-to-tail walls and reduced conductance (dark) at the head-to-head walls. In addition, enhanced conduction is observed at nominally neutral domain wall sections, which is consistent with previous work, where the enhancement was attributed to an accumulation of oxygen interstitials ${ }^{13}$ and the sub-surface domain wall orientation. ${ }^{30}$ To investigate the electronic properties of the charged domain walls in the kilo- to megahertz regime, we perform $\mathrm{AC}_{-\mathrm{CAFM}}{ }^{13}$ scans at the same position. AC-cAFM is a recent spectroscopy method, that allows for probing the $\mathrm{dc}$ response $\left(I_{\mathrm{dc}}^{\text {out }}\right)$ under applied bipolar voltages $\left(V_{\mathrm{ac}}^{\mathrm{in}}\right)$ as a function of frequency (Supporting Information and Figure S1). ${ }^{13} V_{\mathrm{ac}}^{\mathrm{in}}$ describes the amplitude of the bipolar voltage. Figure $1 \mathrm{~b}$ presents the characteristic ACcAFM response of both head-to-head and tail-to-tail domain walls at a frequency $f=0.5 \mathrm{MHz}$. In contrast to previous measurements performed under microwave frequencies, ${ }^{16}$ a pronounced response to the ac voltage is detected at the charged domain walls, clearly separating them from the surrounding domains. In addition, the scan in Figure $1 \mathrm{~b}$ reveals a significant difference in the AC-cAFM response at walls with opposite charge state, showing reduced and enhanced current signals at the head-to-head and tail-to-tail walls, respectively. Thus, the behavior observed in the ACcAFM scan is consistent with the $\mathrm{dc}$ current distribution probed by cAFM (Figure 1a) which is expected to be approached for $f \rightarrow 0 \mathrm{~Hz}$.

A systematic analysis of $I_{\mathrm{dc}}^{\text {out }}$ at charged domain walls as a function of the frequency of the applied ac voltage is presented in Figure $1 \mathrm{c}$ and d. Figure $1 \mathrm{c}$ displays $I_{\mathrm{dc}}^{\text {out }}$ on a logarithmic frequency scale recorded along the solid line indicated in Figure $1 \mathrm{~b}$, featuring a direct comparison of tail-to-tail and head-to-head domain walls with respect to the surrounding domains. At $f=0.1 \mathrm{MHz}, I_{\mathrm{dc}}^{\text {out }}$ at the insulating head-to-head domain wall is suppressed in comparison to the domains,
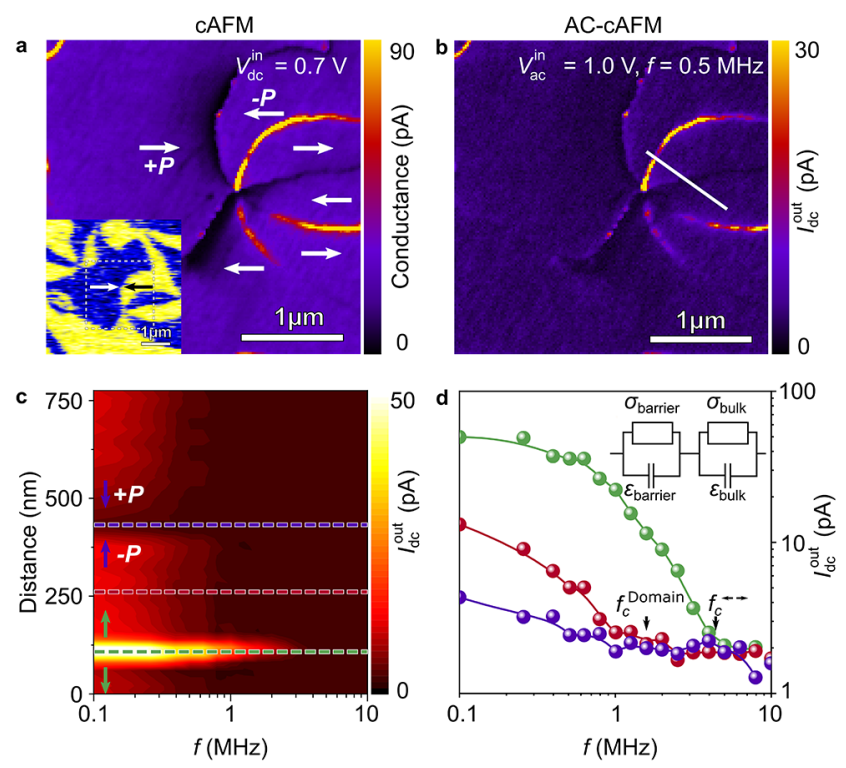

Figure 1. ac response of charged ferroelectric domain walls in $\mathrm{ErMnO}_{3}$. (a) cAFM image displaying reduced and enhanced $\mathrm{dc}$ conductance at head-to-head and tail-to-tail domain walls, respectively. The polarization direction (indicated by the arrows) is obtained from calibrated PFM data, provided in the inset (blue, $+P$; yellow, $-P$ ). (b) AC-cAFM scan taken at the same position as the cAFM image in panel a. (c) Frequency-dependent evolution of the AC-cAFM signal along the solid line in panel b. Pronounced ACcAFM contrast is observed at $f=0.1 \mathrm{MHz}$, vanishing toward increasing frequencies. (d) Local frequency-dependent AC-cAFM response evaluated along the dashed lines in panel $\mathrm{c}$ for a domain, a head-to-head, and tail-to-tail domain wall, indicating different cutoff frequencies, $f_{\mathcal{c}}$ (displayed by arrows) above which the respective signals disappear $\left(f_{\mathrm{c}}^{\leftarrow}>f_{\mathrm{c}}^{\text {Domain }}>f_{\mathrm{c}} \leftarrow\right)$. The equivalent circuit model in the inset allows for relating the frequency drop to the local intrinsic conductivity, ${ }^{13}$ that is, $\sigma_{\text {bulk }}^{\leftarrow}>\sigma_{\text {bulk }}^{\text {Domain }}>\sigma_{\text {bulk }}^{\leftarrow}$ (barrier conductivity, $\sigma_{\text {barrier}}$; barrier permittivity, $\varepsilon_{\text {barrier; }}$ bulk conductivity, $\sigma_{\text {bulk }}$; and bulk permittivity, $\left.\varepsilon_{\text {bulk }}\right)$.

whereas an enhancement of $I_{\mathrm{dc}}^{\text {out }}$ is observed at the tail-to-tail domain wall. With increasing frequency, $I_{\mathrm{dc}}^{\text {out }}$ reveals a steplike feature indicating a relaxation process (Figure $1 \mathrm{~d}$ ). ${ }^{13}$ As indicated by the smaller arrows, a cutoff frequency $f_{c}$ is defined above which $I_{\mathrm{dc}}^{\text {out }}$ reaches a value of less than $1 \%$ of the original value. The cutoff frequency $f_{\mathrm{c}}$ marks a qualitative change in the current-voltage characteristics. Analogous to previous measurements at neutral domain walls in $\mathrm{ErMnO}_{3},{ }^{13}$ the ac response at $f<f_{\mathrm{c}}$ is asymmetric due to the Schottky-like tip-sample contact, leading to a nonzero current signal in AC-cAFM. ${ }^{33,34}$ For $f>f_{\mathcal{c}}$, the AC-cAFM contrast vanishes, indicating symmetric $I(V)$ characteristics. Furthermore, for the conductive tail-to-tail domain wall the cutoff frequency $\left(f_{\mathrm{c}}^{\leftarrow \rightarrow} \sim 4.0 \mathrm{MHz}\right)$ is about four times higher than for the domains $\left(f_{c}^{\text {Domain }} \sim 1.0 \mathrm{MHz}\right)$. Consistent with its reduced dc conductance (Figure 1a), the cutoff frequency of the insulating head-to-head domain wall is below $f_{c}^{\text {Domain }}$. Because of the much lower current signal than for the domains and the tail-to-tail walls, however, it is difficult to unambiguously quantify $\overrightarrow{f_{\mathrm{c}}} \leftarrow$. Thus, we focus on tail-to-tail walls in the later quantitative in-depth analysis.

To rationalize the behavior probed at the charged domain walls, we apply the same equivalent circuit model as used in ref 13 , which is illustrated in the inset to Figure 1d. Here, two $R C$ elements are connected in series. The domains and domain walls are described by a resistor (with conductivity $\sigma_{\text {bulk }}$ ) in 

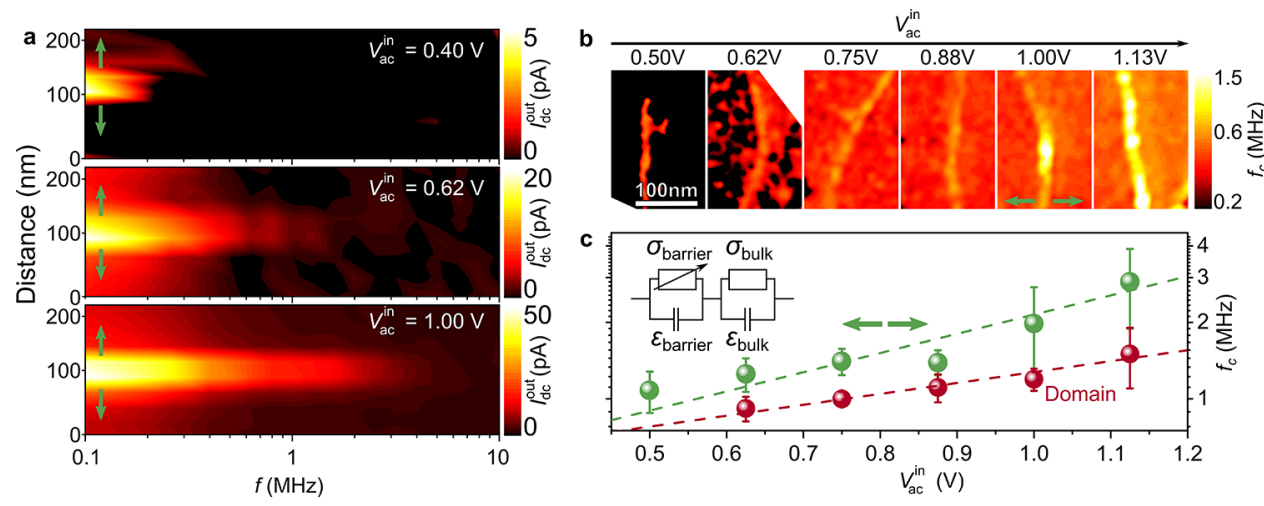

Figure 2. Relation between drive voltage and cutoff frequency. (a) Cross-sectional data showing the frequency dependence of the AC-cAFM response at a negatively charged tail-to-tail domain wall for different voltages. With increasing voltage, $f_{\mathrm{c}}$ shifts to higher frequencies. (b) Spatially resolved measurements of $f_{\mathcal{c}}$ recorded at different tail-to-tail domain walls (see also Figure S2). The data is derived from a series of AC-cAFM scans with logarithmically increasing frequencies by fitting the current decay pixel by pixel as explained in the main text and Figure S3. (c) Comparison of the voltage dependence of the cutoff frequencies measured at tail-to-tail walls and in the surrounding domains. Plotted are the mean values; error bars represent the standard deviation. The dashed lines display a guide to the eye. A nonlinear barrier conductivity is introduced into the equivalent circuit model, as displayed in the inset, which allows for capturing the observed voltage-dependent behavior. ${ }^{42}$

parallel with a capacitor (with permittivity $\varepsilon_{\text {bulk }}$ ). The barrier between tip and sample is described by a barrier conductivity $\left(\sigma_{\text {barrier }}\right)$ connected in parallel with a capacitor (with permittivity $\left.\varepsilon_{\text {barrier }}\right) .^{26,35,36}$ For $f<f_{\mathcal{O}}$ the transport behavior is dominated by the diode-like tip-sample contact, leading to asymmetric current-voltage characteristics and, hence, a pronounced current signal $I_{\mathrm{dc}}^{\text {out }}$ in AC-cAFM. The asymmetric current-voltage characteristics originate from the contact between the probe tip and the p-type semiconducting $\mathrm{ErMnO}_{3}{ }^{23,31}$ Because of the different work function of the tip and $\mathrm{ErMnO}_{3}$, a Schottky barrier is formed at the tip-sample interface, resulting in rectifying current-voltage behavior as discussed, for example, by Wu et al. in ref 34 for the case of ferroelectric domains in $\mathrm{HoMnO}_{3}$. For higher frequencies $(f>$ $f_{\mathrm{c}}$ ), the $I_{\mathrm{dc}}^{\text {out }}$ contrast vanishes, indicating that the tip-sample contact gets short-circuited via the barrier capacitance. ${ }^{37}$ Within our simple equivalent circuit model, the cutoff frequency is defined by the bulk conductivity, $\sigma_{\text {bulk. }}{ }^{13,35,38}$ Here, it is important to note that the measured width of the charged domain walls in our local transport measurements is several tens of nanometers due to spreading of the tip-injected currents as discussed in refs 29 and 39. Thus, to evaluate the cutoff frequency for the domain region, $f_{c}^{\text {Domain }}$, we consider a region $\sim 125 \mathrm{~nm}$ away from the walls. The experimentally determined sequence of cutoff frequencies (Figure 1d), $f_{\mathrm{c}}^{\leftarrow \rightarrow}>$ $f_{\mathcal{c}}^{\text {Pomain }}>f_{\mathcal{c}}^{\rightarrow} \leftarrow$, thus indicates that $\sigma_{\text {bulk }}^{\leftarrow}>\sigma_{\text {bulk }}^{\text {Domin }}>\sigma_{\text {bulk. }}^{\leftarrow}$. This behavior is consistent with dc cAFM measurements (Figure 1a), ${ }^{29}$ where the increase in conductivity at tail-to-tail walls was explained by an enhanced density of mobile holes (majority carriers), which accumulate to screen the negative bound charges at these walls. In contrast, hole depletion occurs to screen the positive bound charges at the head-to-head walls, leading to reduced conductivity relative to the surrounding domains.

To explore the emergence of additional contributions to $f_{c}$ beyond the simplistic equivalent circuit model ${ }^{35,40}$ in Figure $1 \mathrm{~d}$, we next investigate the effect of varying drive voltages on $f_{\text {c }}$.

Voltage-Dependent ac Response at Tail-to-Tail Domain Walls. The effect of varying drive voltage on the cutoff frequency is presented in Figure 2, showing an overview of frequency- and voltage-dependent AC-cAFM measurements for conducting tail-to-tail domain walls (see Figure S2 for complementary cAFM and PFM data). Figure 2a displays spatially resolved data measured along tail-to-tail domain walls with different $V_{\mathrm{ac}}^{\mathrm{in}}$. To avoid possible artifacts caused by repeatedly scanning the same area, ${ }^{41}$ the measurements are performed at different positions on selected walls with comparable dc conductance (see Figure S2 for details). We observe that $f_{\mathrm{c}}$ increases with increasing $V_{\mathrm{ac}}^{\mathrm{in}}$ shifting by more than 1 order of magnitude as $V_{\mathrm{ac}}^{\text {in }}$ is raised from 0.40 to $1.00 \mathrm{~V}$. To systematically analyze the correlation between $f_{\mathrm{c}}$ and $V_{\mathrm{ac}}^{\mathrm{in}}$ we record frequency-dependent AC-cAFM maps for a wider voltage range from which we calculate $f_{\mathrm{c}}$ pixel by pixel as explained in Supporting Information (see Figure S3). Figure $2 \mathrm{~b}$ displays the resulting cutoff-frequency maps for six tail-totail domain walls and the surrounding domains measured at different $V_{\mathrm{ac}}^{\text {in }}$. The mean cutoff frequencies obtained for the domains and domain walls are displayed in Figure $2 c$, and increaseas a function of the applied voltage.

To clarify the origin of the additional drive-voltage dependence revealed by AC-cAFM (Figure 2), we perform complementary voltage-dependent macroscopic spectroscopy experiments on the same single crystal. The frequencydependent loss factor, $\tan \delta$, from $10^{-4}$ to $2 \mathrm{MHz}$ is shown in Figure 3. The voltage and frequency dependence of the dielectric permittivity and the conductivity is displayed in Figure S4. The peak in $\tan \delta$ at $f=5 \times 10^{-2} \mathrm{MHz}$ represents the transition regime between the electrode-sample interface and the intrinsic bulk properties of $\mathrm{ErMnO}_{3} \cdot{ }^{36,43}$ Because of the broadness of the peak, ${ }^{44}$ the electrode-sample interface affects the overall dielectric response even up to much higher frequencies $(f>1 \mathrm{MHz}$, Figure S4). Analogous to the local measurements (Figures 1 and 2), we define a cutoff frequency $f_{\mathrm{c}}\left(\tan \delta\right.$ falls below $25 \%$ of the maximum value, ${ }^{13}$ Figure 3$)$, which takes the broadness of the peak into account. This value $f_{c}$ represents a measure for the frequency at which the contributions from the electrode-sample interface are shortcircuited. In the macroscopic measurements, we find a voltageindependent cutoff frequency $f_{c}=1.3 \mathrm{MHz}$, which agrees with the cutoff frequencies identified for the domains in the local AC-cAFM measurements. Note that the shift of $f_{\mathrm{c}}$ with $V_{\mathrm{ac}}^{\text {in }}$ becomes observable in the local AC-cAFM measurements due to a higher local electric field $(E \approx 40 \mathrm{kV} / \mathrm{cm})$ compared to the electric fields $(E \approx 0.4 \mathrm{kV} / \mathrm{cm})$ used in the macroscopic 


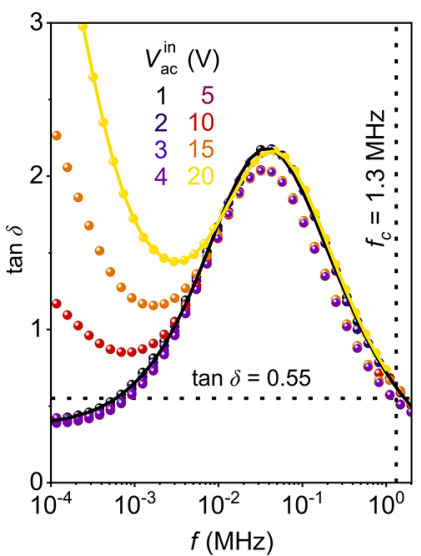

Figure 3. Voltage- and frequency-dependent macroscopic dielectric response. Voltage dependence of the loss factor, $\tan \delta$, as a function of frequency, gained on the same sample as used for the local measurements in Figures 1 and 2. The solid lines represent fits of the experimental data (for $V_{\mathrm{ac}}^{\mathrm{in}}=1 \mathrm{~V}$ and $V_{\mathrm{ac}}^{\mathrm{in}}=20 \mathrm{~V}$ ) utilizing the equivalent circuit model displayed in Figure 1c extended by a frequency-dependent resistance for the bulk as explained previously (see Supporting Information). ${ }^{26,36}$ Analogous to the local measurements (Figures 1 and 2), we define a cutoff frequency, $f_{\mathcal{c}}$ at which the barrier is short-circuited and the bulk response dominates ( $\tan \delta$ falls below $25 \%$ of the maximum value). The identified value of $f_{c}=1.3$ $\mathrm{MHz}$ is in good agreement with the cutoff frequency of the domains found in AC-cAFM (Figure 2c).

measurements. As indicated by the solid lines in Figure 3 and Figure S4, the macroscopic dielectric response can be described via fits using the equivalent circuit model displayed in the inset of Figure 1c (see Supporting Information). The analysis shows that $\sigma_{\text {barrier }}$ increases by more than 1 order of magnitude when $V_{\mathrm{ac}}^{\mathrm{in}}$ is increased from 1 to $20 \mathrm{~V}$, while all other parameters remain almost unchanged.

This leads us to the conclusion that the voltage-dependent AC-cAFM response in Figure 2 originates from the Schottkylike nature of the tip-sample contact. The latter is corroborated by the equivalent circuit fitting of the macroscopic dielectric data, which indicates a substantial voltage-driven barrier lowering (Figure S4), ${ }^{45,46}$ analogous to previous macroscopic measurements on $\mathrm{CaCu}_{3} \mathrm{Ti}_{4} \mathrm{O}_{12}{ }^{47}$ and $\mathrm{BiFeO}_{3}$-based ${ }^{48}$ materials. Thus, the AC-cAFM data gained at the charged domain walls expands previous macroscopic studies on dielectrics to the nanoscale. The voltage dependence of $f_{c}$ (Figure 2c) can be captured by introducing a nonlinear voltage dependence of the barrier conductivity into the equivalent circuit model sketched in the inset to Figure 2c, leading to

$$
f_{\mathrm{c}}\left(V_{\mathrm{ac}}^{\mathrm{in}}\right) \propto \frac{\sigma_{\text {bulk }}+\sigma_{\text {barrier }}\left(V_{\mathrm{ac}}^{\mathrm{in}}\right)}{2 \pi \epsilon_{0} \epsilon_{\text {barrier }}}
$$

In summary, our studies show that the ac characteristics observed at the tail-to-tail domain walls result from their enhanced intrinsic conductivity (Figure 1) in combination with the formation of a voltage-dependent barrier at the electrodewall junction (Figures 2 and 3 ).

Reversible Voltage-Driven Control of the ac Response. The relation between $V_{\mathrm{ac}}^{\mathrm{in}}$ and the response at the tailto-tail domain wall allows for controlling the local electronic transport characteristics. In Figure 4, we demonstrate how the junction between the electrode and the ferroelectric domain wall can be utilized to reversibly switch between uni- and
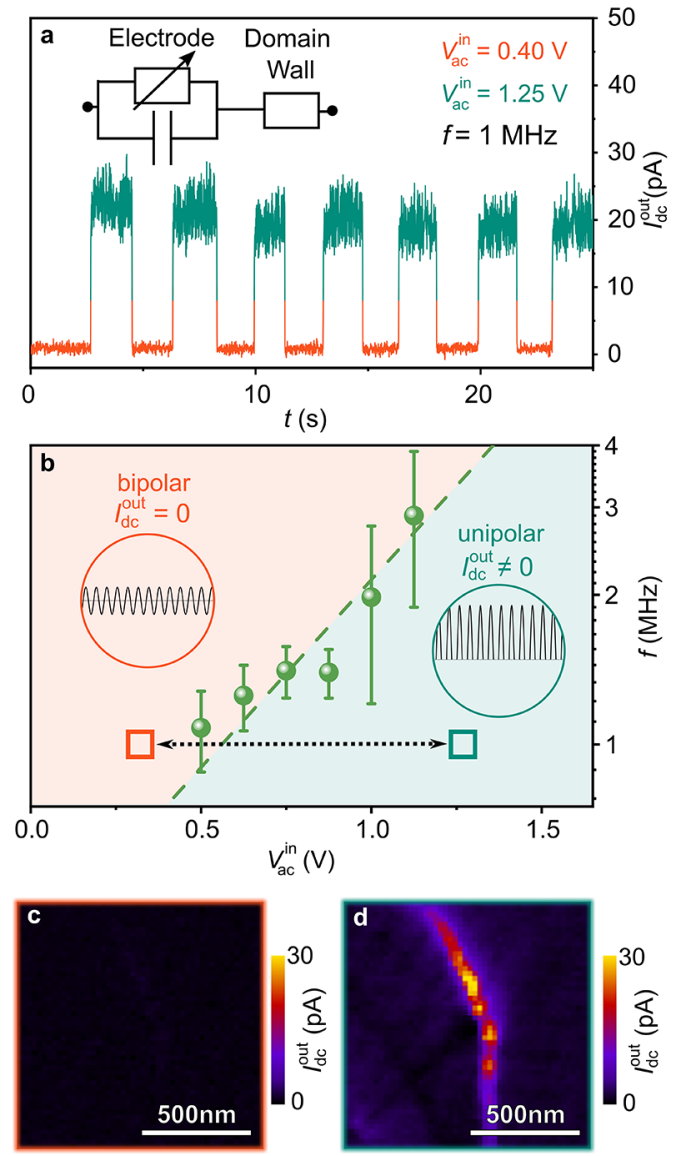

Figure 4. Reversible control of the ac response at tail-to-tail domain walls. (a) AC-cAFM current signal measured with a stationary tip placed on a tail-to-tail domain wall as a function of time over multiple cycles, switching between bipolar (symmetric, $V_{\mathrm{ac}}^{\text {in }}=0.40 \mathrm{~V}, I_{\mathrm{dc}}^{\text {out }}=0$ ) and unipolar (asymmetric, $V_{\mathrm{ac}}^{\text {in }}=1.25 \mathrm{~V}, I_{\mathrm{dc}}^{\text {out }} \neq 0$ ) response at a constant frequency, $f=1 \mathrm{MHz}$. A schematic illustration of a twoterminal ac element emulated by the electrode-wall junction with its equivalent circuit representation ${ }^{42}$ is displayed in the inset. (b) Summary of the electronic response of the ac element in relation to the cutoff frequency and the applied bipolar voltage. The data points and error bars represent $f_{\mathrm{c}}$ (taken from Figure 2c) and mark the transition between a bipolar $\left(I_{\mathrm{dc}}^{\text {out }}=0\right)$ and unipolar $\left(I_{\mathrm{dc}}^{\text {out }} \neq 0\right)$ output signal. This transition between the two distinctly different regimes can either be driven by a change in $V_{\mathrm{ac}}^{\text {in }}(f=$ const.) or, vice versa, by changing $f\left(V_{\mathrm{ac}}^{\mathrm{in}}=\right.$ const $)$. The spatially resolved AC-cAFM image gained for the bipolar and unipolar output of the ac element is displayed in $\mathrm{c}$ and $\mathrm{d}$, respectively (measured at a constant frequency of $f=1 \mathrm{MHz}$ as displayed by the dashed line in panel b).

bipolar output signals. The AC-cAFM data in Figure $4 \mathrm{a}$ is recorded at constant frequency $(f=1 \mathrm{MHz})$ as a function of time, varying the $V_{\mathrm{ac}}^{\text {in }}$ repeatedly between $0.40 \mathrm{~V}$ (orange) and $1.25 \mathrm{~V}$ (green) while keeping the probe tip stationary at the position of the wall. Depending on the applied voltage amplitude, we measure two qualitatively different responses, switching between asymmetric $\left(I_{\mathrm{dc}}^{\text {out }} \neq 0\right)$ and symmetric $\left(I_{\mathrm{dc}}^{\text {out }}=\right.$ $0)$. The two-terminal ac element emulated by the electrodewall junction and the respective equivalent circuit model is sketched in the inset in Figure 4a. The electrode-wall junction responds symmetrically at low $V_{\mathrm{ac}}^{\mathrm{in}}$, whereas an asymmetric response is detected for high $V_{\mathrm{ac}}^{\text {in }}$.

The change in voltage allows reversible switching between unipolar and bipolar output. The dependence of $I_{\mathrm{dc}}^{\text {out }}$ on both 
the applied voltage amplitude and frequency is summarized in Figure $4 \mathrm{~b}$. The data points in Figure $4 \mathrm{~b}$ represent the cutoff frequencies obtained from spectroscopic measurements under constant voltages at a tail-to-tail domain wall (Figure 2). The graph emphasizes the existence of two regimes where the electrode-wall junction exhibits qualitatively different electronic responses. The voltage required to transit between these two regimes can be tuned via the frequency of the input signal. Vice versa, facilitated by the voltage-dependent barrier relaxation (Figure S4c and refs 47 and 48) the cutoff frequency can be selected by adjusting the voltage amplitude of the input signal. Spatially resolved AC-cAFM scans obtained at a tail-totail domain wall at $V_{\mathrm{ac}}^{\mathrm{in}}=0.4 \mathrm{~V}$ and $V_{\mathrm{ac}}^{\mathrm{in}}=1.25 \mathrm{~V}(f=1 \mathrm{MHz})$ are displayed in Figure $4 c$ and $d$, respectively, showing the same switching behavior between a unipolar and bipolar response consistent with the data presented in Figure $2 \mathrm{a}$.

\section{CONCLUSION}

The electronic tunability of the diode-like properties at the electrode-wall junction represents an additional degree of freedom, enabling the design of domain-wall based ac electronic components with ultrasmall feature size. In particular, the involvement of the domain walls ensures that the lateral size is naturally confined with the electronically rectifying area defined by the smallest achievable contact. Application opportunities range from domain-wall based thyrectors that can buffer ripple currents and diodes in transponder circuitry to walls acting as the interconnect between active and passive devices in ac nanoelectronics. In general, the application of charged domain walls in lowfrequency nanoelectronics offers several advantages compared to their neutral counterparts. ${ }^{13}$ In contrast to the neutral walls, which owe their transport properties to the accumulation and/ or depletion of ionic defects, ${ }^{13,30}$ the conduction at charged domain walls is driven by bound polarization charges, that is, an intrinsic mechanism. The latter implies that defect migration and effects from mixed ionic-electric condictivity ${ }^{49}$ play a less important role compared to neutral domain walls, which is important in order to ensure a reversible and deterministic electronic response at the electrode-wall junction. Furthermore, the bound polarization charges can be used as quasi-dopants ${ }^{50}$ to tune the local conductivity and, thereby, engineering the electronic properties of the electrode-wall junction on demand. Our work introduces charged ferroelectric domain walls as versatile building blocks for ac nanoelectronics in the kilo- to megahertz regime, establishing innovative concepts for domain-wall based nanotechnology and the downscaling of electronic ac components in general.

\section{ASSOCIATED CONTENT}

\section{SI Supporting Information}

The Supporting Information is available free of charge at https://pubs.acs.org/doi/10.1021/acs.nanolett.1c03182.

Method Section; schematic description of AC-cAFM; positions of the voltage-dependent AC-cAFM scans for Figure 2; determination of the spatial resolution of the cutoff frequency; voltage- and frequency-dependent macroscopic permittivity and conductivity measurements (PDF)

\section{AUTHOR INFORMATION}

Corresponding Authors

Jan Schultheiß - Department of Materials Science and Engineering, Norwegian University of Science and Technology (NTNU), 7034 Trondheim, Norway; 이잉.org/00000001-7389-1295; Email: jan.schultheiss@ntnu.no

Dennis Meier - Department of Materials Science and Engineering, Norwegian University of Science and Technology (NTNU), 7034 Trondheim, Norway; $\odot$ orcid.org/00000002-8623-6705; Email: dennis.meier@ntnu.no

\section{Authors}

Erik Lysne - Department of Materials Science and Engineering, Norwegian University of Science and Technology (NTNU), 7034 Trondheim, Norway

Lukas Puntigam - Experimental Physics V, University of Augsburg, 86159 Augsburg, Germany

Jakob Schaab - Department of Materials, ETH Zurich, 8093 Zurich, Switzerland

Edith Bourret - Materials Sciences Division, Lawrence Berkeley National Laboratory, Berkeley, California 94720, United States

Zewu Yan - Materials Sciences Division, Lawrence Berkeley National Laboratory, Berkeley, California 94720, United States; Department of Physics, ETH Zurich, 8093 Zurich, Switzerland

Stephan Krohns - Experimental Physics V, University of Augsburg, 86159 Augsburg, Germany

Complete contact information is available at: https://pubs.acs.org/10.1021/acs.nanolett.1c03182

\section{Author Contributions}

J.S. and E.L. recorded the scanning probe microscopy data supervised by D.M. L.P. performed the macroscopic dielectric spectroscopy measurements under supervision of S.K. E.B. and Z.Y. provided the sample. J.S. and D.M. interpreted the data, suggested the device concept, and wrote the manuscript. All authors discussed the results and contributed to the final version of the manuscript.

\section{Notes}

The authors declare no competing financial interest.

\section{ACKNOWLEDGMENTS}

J.S. acknowledges the support of the Alexander von Humboldt Foundation through a Feodor-Lynen research fellowship. D.M. thanks NTNU for support through the Onsager Fellowship Program, the Outstanding Academic Fellow Program, and acknowledges funding from the European Research Council (ERC) under the European Union's Horizon 2020 Research and Innovation Program (Grant Agreement 86691). L.P. and S.K. acknowledge funding of the German Science foundation via the Collaborative Research Center TRR80. E.B. and Z.Y. were supported by the US Department of Energy/Basic Energy Sciences/Division of Materials Sciences and Engineering under Contract DE-AC02-05-CH11231 within the Quantum Materials Program KC2202.

\section{ABBREVIATIONS}

dc, direct current; ac, alternating current; $\mathrm{P}$, polarization; cAFM, conductive atomic force microscopy; PFM, piezoresponse force microscopy. 


\section{REFERENCES}

(1) Catalan, G.; Seidel, J.; Ramesh, R.; Scott, J. F. Domain wall nanoelectronics. Rev. Mod. Phys. 2012, 84 (1), 119-156.

(2) Meier, D. Functional domain walls in multiferroics. J. Phys.: Condens. Matter 2015, 27 (46), 463003.

(3) Nataf, G. F.; Guennou, M.; Gregg, J. M.; Meier, D.; Hlinka, J.; Salje, E. K. H.; Kreisel, J. Domain-wall engineering and topological defects in ferroelectric and ferroelastic materials. Nat. Rev. Phys. 2020, 2 (11), 662.

(4) McCann, E.; Koshino, M. The electronic properties of bilayer graphene. Rep. Prog. Phys. 2013, 76 (5), 056503.

(5) Lembke, D.; Bertolazzi, S.; Kis, A. Single-layer $\mathrm{MoS}_{2}$ electronics. Acc. Chem. Res. 2015, 48 (1), 100-110.

(6) Sato, H.; Bell, C.; Hikita, Y.; Hwang, H. Stoichiometry control of the electronic properties of the $\mathrm{LaAlO}_{3} / \mathrm{SrTiO}_{3}$ heterointerface. Appl. Phys. Lett. 2013, 102 (25), 251602.

(7) Turner, P. W.; McConville, J. P. V.; McCartan, S. J.; Campbell, M. H.; Schaab, J.; McQuaid, R. G. P.; Kumar, A.; Gregg, J. M. Large Carrier Mobilities in $\mathrm{ErMnO}_{3}$ Conducting Domain Walls Revealed by Quantitative Hall-Effect Measurements. Nano Lett. 2018, 18 (10), 6381-6386.

(8) Sharma, P.; Zhang, Q.; Sando, D.; Lei, C. H.; Liu, Y. Y.; Li, J. Y.; Nagarajan, V.; Seidel, J. Nonvolatile ferroelectric domain wall memory. Sci. Adv. 2017, 3 (6), No. e1700512.

(9) Jiang, J.; Bai, Z. L.; Chen, Z. H.; He, L.; Zhang, D. W.; Zhang, Q. H.; Shi, J. A.; Park, M. H.; Scott, J. F.; Hwang, C. S.; Jiang, A. Q. Temporary formation of highly conducting domain walls for nondestructive read-out of ferroelectric domain-wall resistance switching memories. Nat. Mater. 2018, 17 (1), 49-56.

(10) Whyte, J. R.; Gregg, J. M. A diode for ferroelectric domain-wall motion. Nat. Commun. 2015, 6, 7361.

(11) McConville, J. P.; Lu, H.; Wang, B.; Tan, Y.; Cochard, C.; Conroy, M.; Moore, K.; Harvey, A.; Bangert, U.; Chen, L. Q.; Gruverman, A.; Gregg, J. M. Ferroelectric Domain Wall Memristor. Adv. Funct. Mater. 2020, 30, 2000109.

(12) Mundy, J. A.; Schaab, J.; Kumagai, Y.; Cano, A.; Stengel, M.; Krug, I. P.; Gottlob, D. M.; Doganay, H.; Holtz, M. E.; Held, R.; Yan, Z.; Bourret, E.; Schneider, C. M.; Schlom, D. G.; Muller, D. A.; Ramesh, R.; Spaldin, N. A.; Meier, D. Functional electronic inversion layers at ferroelectric domain walls. Nat. Mater. 2017, 16 (6), 622627.

(13) Schaab, J.; Skjærvø, S. H.; Krohns, S.; Dai, X. Y.; Holtz, M. E.; Cano, A.; Lilienblum, M.; Yan, Z. W.; Bourret, E.; Muller, D. A.; Fiebig, M.; Selbach, S. M.; Meier, D. Electrical half-wave rectification at ferroelectric domain walls. Nat. Nanotechnol. 2018, 13 (11), 10281034

(14) Bednyakov, P. S.; Sturman, B. I.; Sluka, T.; Tagantsev, A. K.; Yudin, P. V. Physics and applications of charged domain walls. Npj. Comput. Mater. 2018, 4, 65.

(15) Jiang, A. Q.; Zhang, Y. Next-generation ferroelectric domainwall memories: principle and architecture. NPG Asia Mater. 2019, 11 (1), $1-5$.

(16) Wu, X. Y.; Petralanda, U.; Zheng, L.; Ren, Y.; Hu, R. W.; Cheong, S. W.; Artyukhin, S.; Lai, K. J. Low-energy structural dynamics of ferroelectric domain walls in hexagonal rare-earth manganites. Sci. Adv. 2017, 3 (5), No. e1602371.

(17) Wu, X.; Du, K.; Zheng, L.; Wu, D.; Cheong, S.-W.; Lai, K. Microwave conductivity of ferroelectric domains and domain walls in a hexagonal rare-earth ferrite. Phys. Rev. B: Condens. Matter Mater. Phys. 2018, 98 (8), No. 081409.

(18) Lummen, T. T. A.; Leung, J.; Kumar, A.; Wu, X.; Ren, Y.; VanLeeuwen, B. K.; Haislmaier, R. C.; Holt, M.; Lai, K. J.; Kalinin, S. V.; Gopalan, V. Emergent Low-Symmetry Phases and Large Property Enhancements in Ferroelectric $\mathrm{KNbO}_{3}$ Bulk Crystals. Adv. Mater. 2017, 29 (31), 1700530.

(19) Tselev, A.; Yu, P.; Cao, Y.; Dedon, L. R.; Martin, L. W.; Kalinin, S. V.; Maksymovych, P. Microwave ac conductivity of domain walls in ferroelectric thin films. Nat. Commun. 2016, 7, 11630.
(20) Huang, Y.-L.; Zheng, L.; Chen, P.; Cheng, X.; Hsu, S.-L.; Yang, T.; Wu, X.; Ponet, L.; Ramesh, R.; Chen, L.-Q.; Artyukhin, S.; Chu, Y.-H.; Lai, K. Unexpected Giant Microwave Conductivity in a Nominally Silent $\mathrm{BiFeO}_{3}$ Domain Wall. Adv. Mater. 2020, 32 (9), 1905132.

(21) Gu, Z.; Pandya, S.; Samanta, A.; Liu, S.; Xiao, G.; Meyers, C. J.; Damodaran, A. R.; Barak, H.; Dasgupta, A.; Saremi, S. Resonant domain-wall-enhanced tunable microwave ferroelectrics. Nature 2018, 560 (7720), 622-627.

(22) Prosandeev, S.; Yang, Y. R.; Paillard, C.; Bellaiche, L. Displacement Current in Domain Walls of Bismuth Ferrite. Npj. Comput. Mater. 2018, 4, 8 .

(23) Subba Rao, G.V.; Wanklyn, B.M.; Rao, C.N.R. Electrical Transport in Rare Earth Ortho Chromites, Ortho Manganites and Ortho Ferrites. J. Phys. Chem. Solids 1971, 32 (2), 345-358.

(24) Skjærvø, S. H.; Wefring, E. T.; Nesdal, S. K.; Gaukas, N. H.; Olsen, G. H.; Glaum, J.; Tybell, T.; Selbach, S. M. Interstitial oxygen as a source of p-type conductivity in hexagonal manganites. Nat. Commun. 2016, 7, 13745.

(25) Schoenherr, P.; Shapovaloy, K.; Schaab, J.; Yan, Z.; Bourret, E. D.; Hentschel, M.; Stengel, M.; Fiebig, M.; Cano, A.; Meier, D. Observation of Uncompensated Bound Charges at Improper Ferroelectric Domain Walls. Nano Lett. 2019, 19 (3), 1659-1664.

(26) Ruff, A.; Li, Z.; Loidl, A.; Schaab, J.; Fiebig, M.; Cano, A.; Yan, Z.; Bourret, E.; Glaum, J.; Meier, D.; Krohns, S. Frequency dependent polarisation switching in h-ErMnO 3 . Appl. Phys. Lett. 2018, 112 (18), 182908.

(27) Van Aken, B. B.; Palstra, T. T. M.; Filippetti, A.; Spaldin, N. A. The origin of ferroelectricity in magnetoelectric $\mathrm{YMnO}_{3}$. Nat. Mater. 2004, 3 (3), 164-170.

(28) Fennie, C. J.; Rabe, K. M. Ferroelectric transition in $\mathrm{YMnO}_{3}$ from first principles. Phys. Rev. B: Condens. Matter Mater. Phys. 2005, 72 (10), 100103.

(29) Meier, D.; Seidel, J.; Cano, A.; Delaney, K.; Kumagai, Y.; Mostovoy, M.; Spaldin, N. A.; Ramesh, R.; Fiebig, M. Anisotropic conductance at improper ferroelectric domain walls. Nat. Mater. 2012, 11 (4), 284-288.

(30) Schultheiß, J.; Schaab, J.; Småbråten, D. R.; Skjærvø, S. H.; Bourret, E.; Yan, Z.; Selbach, S. M.; Meier, D. Intrinsic and extrinsic conduction contributions at nominally neutral domain walls in hexagonal manganites. Appl. Phys. Lett. 2020, 116 (26), 262903.

(31) Choi, T.; Horibe, Y.; Yi, H. T.; Choi, Y. J.; Wu, W. D.; Cheong, $\mathrm{S}$. W. Insulating interlocked ferroelectric and structural antiphase domain walls in multiferroic $\mathrm{YMnO}_{3}$. Nat. Mater. 2010, 9 (3), 253258.

(32) Wu, W.; Horibe, Y.; Lee, N.; Cheong, S. W.; Guest, J. R. Conduction of Topologically Protected Charged Ferroelectric Domain Walls. Phys. Rev. Lett. 2012, 108 (7), 077203.

(33) Pintilie, L.; Alexe, M. Metal-ferroelectric-metal heterostructures with Schottky contacts. I. Influence of the ferroelectric properties. J. Appl. Phys. 2005, 98 (12), 124103.

(34) Wu, W.; Guest, J. R.; Horibe, Y.; Park, S.; Choi, T.; Cheong, S. W.; Bode, M. Polarization-Modulated Rectification at Ferroelectric Surfaces. Phys. Rev. Lett. 2010, 104 (21), 217601.

(35) Lunkenheimer, P.; Krohns, S.; Riegg, S.; Ebbinghaus, S. G.; Reller, A.; Loidl, A. Colossal dielectric constants in transition-metal oxides. Eur. Phys. J.: Spec. Top. 2009, 180, 61-89.

(36) Ruff, E.; Krohns, S.; Lilienblum, M.; Meier, D.; Fiebig, M.; Lunkenheimer, P.; Loidl, A. Conductivity Contrast and Tunneling Charge Transport in the Vortexlike Ferroelectric Domain Patterns of Multiferroic Hexagonal $\mathrm{YMnO}_{3}$. Phys. Rev. Lett. 2017, 118 (3), 036803.

(37) Lunkenheimer, P.; Bobnar, V.; Pronin, A. V.; Ritus, A. I.; Volkov, A. A.; Loidl, A. Origin of apparent colossal dielectric constants. Phys. Rev. B: Condens. Matter Mater. Phys. 2002, 66 (5), 052105 .

(38) Chasin, A.; Volskiy, V.; Libois, M.; Myny, K.; Nag, M.; Rockelé, M.; Vandenbosch, G. A.; Genoe, J.; Gielen, G.; Heremans, P. An 
integrated a-IGZO UHF energy harvester for passive RFID tags. IEEE Trans. Electron Devices 2014, 61 (9), 3289-3295.

(39) Schaab, J.; Cano, A.; Lilienblum, M.; Yan, Z. W.; Bourret, E.; Ramesh, R.; Fiebig, M.; Meier, D. Optimization of Electronic Domain-Wall Properties by Aliovalent Cation Substitution. Adv. Electron. Mater. 2016, 2 (1), 1500195.

(40) Biškup, N.; De Andrés, A.; Martinez, J.; Perca, C. Origin of the colossal dielectric response of $\operatorname{Pr}_{0.6} \mathrm{Ca}_{0.4} \mathrm{MnO}_{3}$. Phys. Rev. B: Condens. Matter Mater. Phys. 2005, 72 (2), 024115.

(41) Wang, X. Y.; Yang, D. N.; Zhang, H. M.; Song, C. Y.; Wang, J.; Tan, G. T.; Zheng, R. K.; Dong, S.; Cheong, S. W.; Zhang, J. X. Anisotropic resistance switching in hexagonal manganites. Phys. Rev. B: Condens. Matter Mater. Phys. 2019, 99 (5), 054106.

(42) Semple, J.; Georgiadou, D. G.; Wyatt-Moon, G.; Gelinck, G.; Anthopoulos, T. D. Flexible diodes for radio frequency (RF) electronics: a materials perspective. Semicond. Sci. Technol. 2017, 32 (12), 123002.

(43) Puntigam, L.; Schultheiß, J.; Strinic, A.; Yan, Z.; Bourret, E.; Altthaler, M.; Kezsmarki, I.; Evans, D. M.; Meier, D.; Krohns, S. Insulating improper ferroelectric domain walls as robust barrier layer capacitors. J. Appl. Phys. 2021, 129, 074101.

(44) Emmert, S.; Wolf, M.; Gulich, R.; Krohns, S.; Kastner, S.; Lunkenheimer, P.; Loidl, A. Electrode polarization effects in broadband dielectric spectroscopy. Eur. Phys. J. B 2011, 83 (2), $157-165$.

(45) Rideout, V. A review of the theory, technology and applications of metal-semiconductor rectifiers. Thin Solid Films 1978, 48 (3), 261-291.

(46) Sze, S.; Crowell, C.; Kahng, D. Photoelectric determination of the image force dielectric constant for hot electrons in Schottky barriers. J. Appl. Phys. 1964, 35 (8), 2534-2536.

(47) Krohns, S.; Lunkenheimer, P.; Ebbinghaus, S. G.; Loidl, A. Colossal dielectric constants in single-crystalline and ceramic $\mathrm{CaCu}_{3} \mathrm{Ti}_{4} \mathrm{O}_{12}$ investigated by broadband dielectric spectroscopy. $J$. Appl. Phys. 2008, 103 (8), 084107.

(48) Morozov, M. I.; Einarsrud, M.-A.; Grande, T. Atmosphere controlled conductivity and Maxwell-Wagner relaxation in $\mathrm{Bi}_{0.5} \mathrm{~K}_{0.5} \mathrm{TiO}_{3}-\mathrm{BiFeO}_{3}$ ceramics. J. Appl. Phys. 2014, 115 (4), 044104.

(49) Bencan, A.; Drazic, G.; Ursic, H.; Makarovic, M.; Komelj, M.; Rojac, T. Domain-wall pinning and defect ordering in $\mathrm{BiFeO}_{3}$ probed on the atomic and nanoscale. Nat. Commun. 2020, 11 (1), 1762.

(50) Crassous, A.; Sluka, T.; Tagantsev, A. K.; Setter, N. Polarization charge as a reconfigurable quasi-dopant in ferroelectric thin films. Nat. Nanotechnol. 2015, 10 (7), 614-618. 